\title{
Genes and life-style factors in BELFAST nonagenarians: Nature, Nurture and Narrative
}

\author{
Jennifer Nicola M. Rea • Ashley Carvalho • \\ Susan E. McNerlan • H. Denis Alexander • \\ Irene Maeve Rea
}

Received: 20 January 2015/Accepted: 4 March 2015/Published online: 14 March 2015

(C) The Author(s) 2015. This article is published with open access at Springerlink.com

\begin{abstract}
Understanding how to 'Age Longer and Age Well' is a priority for people personally, for populations globally and for government policy. Nonagenarians are the oldest members of our societies and survivors of their generation. Approximately $10 \%$ of nonagenarians reach 90 years and beyond in good condition and seem to have a combination of both age-span and health-span. But what are the factors which help people reach their ninetieth birthday and beyond in good condition? Are they genetics, as in 'nature', or do they depend on 'nurture' and are related to environment, or are both factors inextricably intertwined within the concept of behavioural genetics? Nonagenarians have rich life experiences that can teach us much about ageing well; they are reservoirs of genetic, life-style and behavioural information which
\end{abstract}

J. N. M. Rea

Research Department Primary Care and Population

Health, University College London, London, UK

A. Carvalho · I. M. Rea ( $\square)$

School of Medicine, Dentistry and Biomedical Science, Queens University Belfast, Belfast, Northern Ireland, UK e-mail: i.rea@qub.ac.uk

\section{S. E. McNerlan}

Regional Cytogenetics Laboratory, Belfast Health and Social Care Trust, Belfast, Northern Ireland, UK

H. D. Alexander · I. M. Rea

School of Biomedical Science, University of Ulster, Coleraine, Northern Ireland, UK can help dissect out how to live not only longer but better. Personal family history and narrative are powerful tools that help to determine familial traits, beliefs and social behaviours and when used in parallel with new biotechnology methods inform and elaborate causality. Here we present themes and insights from personal narrative enquiry from nonagenarian participants from the Belfast Elderly Longitudinal Freeliving Ageing STudy (BELFAST) about factors they consider important for good quality ageing and relate these insights to the emerging genetics and life-style evidence associated with healthy longevity.

Keywords BELFAST nonagenarians - Genes · Life-style $\cdot$ Nurture $\cdot$ Nature $\cdot$ Narrative

\section{Introduction}

Mixed method research, using both quantitative and qualitative aspects, has the potential to ask contrasting and distinctive questions about the interface between the scientific and social world, and to gain understanding about how the evidence from biological and life sciences can be acted out in the real world of individual beliefs and behaviours. Those differences-and relationships between the interdisciplinary fields of life sciences and those of social science, arts and humanities-help to bring the relevance of science into the socio-economic world 
of everyday life, because as a recent editorial in Nature argues 'science doesn't advance far if we don't understand the dynamics of behaviour, attitude and organisation' (Editor 2015).

Present day social science is increasingly recognising the validity of the nonagenarian insights which are telling us powerful messages about their ageing and are directly in line with present-day public health campaigns-encouraging populations to adopt better lifestyles and behaviours in order to make for a healthier life course.

Therefore in the BELFAST nonagenarian study, it was of interest to identify what range of behaviours, interventions and self-management strategies had supported and contributed to the BELFAST 'elite' nonagenarian's combination of age-span and healthspan, and which exemplified the Perl 'escaper' phenotype (Evert et al. 2003). Through narrative enquiry, we aimed to get a sense of the nonagenarian knowledge and engagement with their own good quality ageing, in terms of life-style and behaviours.

Here we discuss the main themes which the nonagenarian themselves identified as important pointers in their age-span and health-span, relate these to some present day evidence about genes and life style interactions related to longevity and discuss how the combined evidence supports everyone adopting better ageing strategies.

\section{Subjects and methods}

In the BELFAST study, 90 year old subjects, who were 'very good' for their age, also called 'elite' and met the Senieur protocol (Lightart et al. 1984), were recruited through their General Practitioners, from the Greater Belfast area. Subjects willing to enrol, were community-living, mobile, and mentally competent (mini mental score examination $>26 / 30$ ), (Folstein et al. 1975) and gave written consent. Briefly, subjects gave blood samples for DNA and other laboratory variables, responded to nutrition, life style and medical history questionnaires, blood pressure and anthropometric measurements (Rea et al. 2009) and provided self-directed narrative life-stories (Ganzevoort and Bouwer 2007) together with structured questions (Rea and Rea 2011; Rea Maeve 2013). Ethical permission for the Belfast Elderly Longitudinal Free-Living Ageing STudy (BELFAST) studies was given by Research Ethics Committee Northern Ireland (ORECNI), 08/NIR03/42 and by The Queens University Belfast.

\section{Nonagenarians narrative insights about their longevity}

Using grounded theory and qualitative analysis (Glaser and Strauss 1967; Strauss and Corbin 1990), five key findings and insights were identified by nonagenarians themselves as important in their long lives and good quality health (Rea and Rea 2011; Rea Maeve 2013).

The five main themes were identified by BELFAST nonagenarians as important factors in their longevity included (1) genes (2) diet (3) good health (4) physical activity (5) social networks and resilience. Each will be discussed briefly in the context of some of the present day evidence relating to longevity.

\section{Genes and longevity}

Apolipoprotein E gene (ApoE)

Many nonagenarians considered that the genes within their extended families were important factors contributing to their long lives.

The ApoE gene is one of the most important and consistent genes identified with respect to both healthy longevity and age-related disorders. Age-related diseases such as Alzheimers, and vascular dementia (Corder et al. 1993; Licastro et al. 2007; Chuang et al. 2010) and cardiovascular risk (Eichner et al. 2002; Ilveskoski et al. 1999) have been regularly associated with the ApoE gene and particularly the ApoE4 allele which tracks with higher lipoprotein values (Bennet et al. 2007). In parallel studies ApoE has also been identified as the single most important gene associated with 'healthy longevity' in a host of individual studies including locally in BELFAST nonagenarians (Bennati et al. 2010; Rea et al. 2001), several meta-analyses and most recently in the European Genetics of Healthy Ageing (GeHA) nonagenarian sibling cohort where it is closely related to the TOMM 40 gene location (Beekman et al. 2013; Deelan et al. 2011; Deelen et al. 2014), which is mostly associated with the ApoE4 variant (Brooks-Wilson 2013). Early research by 
Schachter et al. (1994) had identified ApoE2 allele as being more frequent in French nonagenarians and this finding has been confirmed in other studies where not only was ApoE2 allele frequency accentuated but carriage of the ApoE4 allele was reduced in nonagenarian and centenarians cohorts (Frisoni et al. 2001; Kervinen et al. 1994; Cauley et al. 1993; Eggertsen et al. 1993; Corder et al. 1996). In keeping with this hypothesis, BELFAST nonagenarians, showed a fall in ApoE4 allele frequency reduced from $16 \%$ in $<65$ year olds from the same geographical area, enlisted for the MONItoring of CArdiovascular (MONICA) project, down to $8 \%$ in BELFAST nonagenarians. There was a reciprocal change in ApoE2 allele frequency rising to $12 \%$ in nonagenarians compared to $8 \%$ in $<65$ year old MONICA local subjects (Rea et al. 2001). One of the main explanations for these findings has been the association of ApoE4 carriage with a more risky lipoprotein profile (Bennet et al. 2007) which likely contributed to earlier mortality through vascular disease affecting the brain and cardiovascular system. Hence the frequency of the ApoE4 allele showed attrition in the oldest healthiest nonagenarian and centenarian cohorts.

Added to this disease risk profile of the ApoE4 allele, the GeHA study of nonagenarian siblings (Beekman et al. 2013), clearly replicated the known stratification of ApoE frequencies North-South in Europe with higher population frequencies of ApoE4 in Northern Europe compared to Southern countries. This finding also appeared to track with the increased susceptibility of cardiovascular disease risk. It is also known that ApoE4 frequency is much lower in some areas of the world (Corbo and Scacchi 1999) with a baseline frequency reported of $10 \%$ at age 20 falling to $4 \%$ in the $>1990$ s in Han Chinese, suggesting a similar attrition with age for ApoE4 allele but from a lower baseline population frequency (Jian-Gang et al. 1998; Lu et al. 2014). Again there is the consideration that this lower ApoE population frequency may associate with a lower baseline frequency and risk of cardiovascular disease early mortality.

\section{Diet, genes and life-style}

ApoE and life-style factors

The allelic variants of ApoE are associated with increasing cholesterol and lipid profile in the range
E4 $>$ E3 $>$ E2 so that E4 carriers have higher lipoprotein fractions and associated increased risk of cardiovascular disease, dementia and stroke, compared to E2 carriers (Bennet et al. 2007). BELFAST nonagenarians who carried the ApoE4 allele showed higher serum cholesterol values compared to those who carried the ApoE2 allele in keeping with the finding of increased vascular-related risk and attrition effect on the ApoE4 gene pool in 90 year old survivors. Changes in modern-day lifestyle have been argued to be important in the present-day and atherosclerotic-related risk for ApoE function in Western populations who have adopted higher fat diets and a less physical life-style. Raichlen and Alexander 2014 have argued and provide evidence that ApoE4, which is the ancestral variant, is negatively affected by smoking, high fat diet and a sedentary life-style but can be modulated by appropriate excise and diet regimes similar to those which were part of the life-style of our ancestors. Emphasising the same theme, Mattson (2012) argues that the fast food and the doorstep pizza delivery was not part of the lifestyle of our ancestors who had to spend much more physical energy in obtaining their daily energy needs (Mattson 2012).

One of the biggest conundrums is the association of the ApoE gene with both longevity and age-related disease (Perls 2002; Zhang et al. 2008). Does this suggest that the life style factors which BELFAST nonagenarians or the Perl 'escaper' phenotype (Evert et al. 2003) have adopted are instrumental in delaying age-related diseases and vascular risk (Hagberg et al. 2000; Masson et al. 2003; Ordovas 2008) and that through a combination of behavioural genetics and life-style factors, 'elite' nonagenarians intuitively chose the different life experiences, diets and lifestyles best suited to them (Gibney and Walsh 2013)?

\section{TCF7L2 gene and Mediterranean diet}

The relationship between the TCF7L2 and the Mediterranean diet is one of the most recognised gene/diet interactions (Corella et al. 2013). Strict compliance with the Mediterranean diet can modulate the damaging effects of the T risk allele which is an important risk factor for development of diabetes.

Importantly, in the context of longevity, Garagnani et al. (2013) argued that centenarians could be used as 'super controls' to assess the biological significance of 
genetic markers for age-related diseases, for Type 2 diabetes and the TCF7L2 genotypes. The group reported a marked reduced frequency of the diabetes-related risk $\mathrm{T}$ allele of TCF7L2 in their super centenarian controls, but an enrichment of the homozygous $\mathrm{CC}$ genotype, suggesting that the $\mathrm{CC}$ genotype could be a strong protective variant, at least in Italian centenarians likely to have been exposed to a Mediterranean diet.

\section{Good health and immunity}

Cytokine genes

Cytokine genes control cytokines, which in turn drive the immune response (McNerlan et al. 2009). Together they orchestrate and maintain the immune system thorough out life and seem likely to have an important role in good quality ageing and longevity. Cytokine polymorphisms have functional effects which determine serum cytokine responsiveness to danger signals, including infections, cancerous cells, toxins, diet and exercise (Ross et al. 2003; Rea et al. 2006). Considering the continual pressures from internal and external stressors, the immune system's adaptability is being constantly shaped and re-shaped thorough out life. It seems likely that there are different cytokine gene polymorphisms and phenotypes, some of which contribute to the age-related disease phenotype and are perhaps associated with a strongly pro-inflammatory response, which could contribute to survival from childhood infections, but might drive immune activation with a predilection to age-related disease later in life (Rea et al. 2006), while conversely another cytokine phenotype could be associated with an accentuated anti-inflammatory profile, a more modulated immune response to mid life 'stressors' and/or age-related disease and contribute to a cytokine profile, better shaped to facilitate longevity (Lio et al. 2002).

\section{IL-6 and Mediterranean diet}

The IL-6 gene is called the gerontologist cytokine (Ershler 1993) and was considered a likely candidate in longevity and good quality ageing, because most age-related diseases are associated with increases in serum IL-6. Subjects homozygous for the risk allele
(C) of the-174 G/C IL-6 polymorphism have higher levels of IL-6 than do G genotype carriers and a demonstrably increased risk from cardiovascular disease (Spoto et al. 2014). It was therefore argued that the G-allele-carriers might be more common in those who became nonagenarians or centenarians. In the local BELFAST nonagenarian cohort, the frequency of homozygous IL- $6 \mathrm{G}$ allele showed some attrition in very aged persons but was insufficiently powered (Rea et al. 2003). A subsequent European meta-analysis demonstrated a shift in IL-6-G-allele frequencies North/South but showed no significant association with longevity in the combined aged-cohorts (Di Bona et al. 2009). However a separate analysis of southern European centres showed an increased odds ratio for the G-allele and longevity (Di Bona et al. 2009). Here it was postulated that the IL-6 gene polymorphisms could be modulated by the Mediterranean diet as has been noted in cellular studies (Mena et al. 2009; Camargo et al. 2010) with the Mediterranean diet contributing to differences between Northern and Southern European incidence, and mortality from vascular disease (Fung et al. 2009; de Lorgeril and Salen 2006).

Autoimmune disease and Mediterranean diet

Similarly there is increasing evidence that autoimmune diseases such as rheumatoid arthritis or lupus or ulcerative colitis may be modulated by compliance with a Mediterranean type diet (Skoldstam et al. 2003; McKellar et al. 2007) which appears to reduce the inflammatory profile of disease through mechanisms which are considered to be mediated through downregulation of the NFkB pathway, which stands at the cross-roads of the cellular inflammatory cascade (Lawrence 2009).

Diet, exercise and anti-oxidant status

In exercise-related stress there is evidence that antioxidant capacity is important in reducing post-exercise stress (Radak et al. 2008) and that a careful control of anti-oxidant flux through diet may help to reduce damaging post-exercise cytokine responses (Lamina et al. 2013). While a moderate amount of exercise is considered good (Cobley et al. 2014; Radak et al. 2005) endurance and ultra marathon type sports can contribute to long-term damage and immune 
activation which does not settle between episodes and the positive and beneficial effects of 'hormesis' are lost (Lushchak 2014).

\section{Genetics and NK cells}

Natural Killer cell (NK) populations have been found to be increased with increasing age (Miyaji et al. 1997; Sansoni et al. 1993; McNerlan et al. 1998). NK cells, together with related NKT subsets, the killer cell immunoglobulin-like receptor (KIR) receptor-genecomplexes and associated cytokine profiles, are highly important in effective in patrolling, controlling and protecting our immune landscape thorough out life from viruses, cancerous and damaged cells of all kinds (Peralbo et al. 2007; Rea et al. 2013. Their roles and interactions are likely to be important in maintaining immune integrity in people who live successfully into their 1990s and beyond (Mocchegiani and Malavolta 2004) and fit the criteria of the Perl 'escaper' model of successful ageing (Evert et al. 2003). The KIR genes control the functions of NK cells through A or B haplotypes, with A having a more inhibitory role on NK function, compared to the more activating role of $\mathrm{B}$ the KIR haplogroup. BELFAST nonagenarians grouped by $\mathrm{A}$ and B KIR haplotype showed a predominantly $\mathrm{B}$ haplogroup inflammatory cytokines profile for B haplogroup carriers but increased numbers of NK cells for nonagenarians carrying the KIR A haplogroup (Rea et al. 2013; Maxwell et al. 2004). These differences in KIR haplotype effects may be important in explaining the longevity phenotype with the increased number of NK and NKT-related cells found in nonagenarians (McNerlan et al. 1998; Peralbo et al. 2007) or conversely with the proinflammatory background found with increasing age or inflamm-ageing (Franceschi et al. 2000).

NK cells and diet

NK cells do appear to have a relationship with nutrition and diet. Their cytolytic activity of NK receptors have shown to be upregulated by nutrition measures including Vitamin D and anthropometric markers (von Essen et al. 2010; Cantorna et al. 2012). Scientists have found that vitamin D is crucial to activating our immune defences and that without sufficient intake of the vitamin D the killer cells of the immune system are not able to react to fight off serious infections in the body (Al-Jaderi and Maghazachi 2013). In preliminary work, there is a small negative relationship between NK cell number and BMI in BELFAST nonagenarians (Rea et al. 2013) which replicates findings between BMI and anthropometric measurements identified previously in Italian aged cohorts (Mariani et al. 1999; Ravaglia et al. 2000). This finding could be important in relation to the obesity paradox which has described improved outcomes from life-threatening serious infections and major surgical procedures in elderly people with higher BMIs (Hogue et al. 2009; Kuperman et al. 2013).

\section{Physical activity}

Many of the nonagenarian cohort reported continuing to be physically active thorough out their lives. The nonagenarians are no longer alone in their belief that one of the secrets of survivorship is working hard and maintaining physical activities. The Cambridge University study of 334,000 people found that even a modest amount of physical activity prolonged life (Ekelund et al. 2015), with similar findings in another large pooled cohort analysis (Moore et al. 2012). A prospective observational study involving almost half a million Taiwanese reported that being active for as little as 15 min a day can add as much as 3 years to the length of life (Wen et al. 2011) and a major study in USA has further shown that maintaining active exercise was an important contributor to good quality ageing for male physicians, who reached 90 years of age in good health (Yates et al. 2008). In a recent systematic review of physical activity and healthy ageing including cognitive function, there were clear outcome findings suggesting that late-life physical activity is beneficial for cognitive function in elderly people, with three studies reporting a dose-response relationship between physical activity and cognition (Carvalho et al. 2014). Although mechanisms remain to be fully identified, brain-derived neurotrophic factor (BDNF) is known to be heavily involved in the differentiation, extension, and survival of neurons in the hippocampus, cortex and cerebellum during brain development (Neeper et al. 1995; Vaynmana et al. 2006), and some animal models (Rhyu et al. 2010; Patten et al. 2013; Merkley et al. 2014) followed by clinical studies, demonstrate that BDNF level is 
associated with hippocampal volume and with aerobic exercise (Pang and Hannan 2013; Lojovich 2010; Muscari et al. 2010; Erickson et al. 2011).

These studies all add to the accumulating evidence that says that exercise is good for everyone (Ploughman 2008), irrespective of age and may also keep our brains in sharper function by stimulating and maintaining neurogenesis.

\section{Genetic and molecular mechanisms of resilience}

Social networks and resilience

Successful ageing is considered to be more than the "absence of disease and maintenance of high functioning" (Baltes and Baltes 1990), but involves the active engagement in everyday social activities.

The value of good social networks and family interactions has been previously described as important in good quality ageing (Evert et al. 2003), with the number of social interactions daily and inclusion in family networks making for good mental health and social well-being (Rowe and Kahn 1997).

Although much remains to be clarified, the mechanisms through which stress can affect mental and physical health and resilience are becoming clearer. Excessive glucocorticoids (GCs) released after early life stress exposure seem to cause longlasting destabilisation of the stress hormone system which in turn increases risk for later psychiatric disorders. These findings follow on the early work in animal studies whereby poor maternal care was shown to cause dysregulation of the HPA axis in rodents with altered transcription of the GC receptor gene (Meaney 2001). Absence of early nurturing, through licking behaviour in rodent models, led to changes in the promoter of the GC receptor NR3C1 gene (Meaney and Szyf 2005), with similar changes demonstrated in people with bipolar disorders and later life stress which correlated with frequent general practice attendance (Furukawa et al. 1999; Glaser et al. 2006; Francis et al. 1999).

Around this theme, nonagenarians reported 'being happy', 'always cheerful', 'never melancholy' and having a contentment with a 'rich life' and family relationships 'thank God I have such good children'. Social networks and supportive relationships are known to buffer the effects of stressful life events such as bereavement, deteriorating health and loss of autonomy (Northern Arizona University 1998) and to mitigate negative feelings and emotions (Rentoul 1997).

\section{Summary comments}

The themes emerging from collected life stories collected of 'elite' nonagenarians provide some insights into why nonagenarians believe they have lived so long and well. Their narratives and answers are an adjunct to a whole range of ongoing scientific genetic enquiry using innovative new technologies and bioinformatics to search out the genetics and lifestyle patterns of longevity. Personal insights from nonagenarians themselves provide important information about beliefs, behaviours and social circumstances which further enrich our understanding of the ageing-well phenotype (Baltes and Baltes 1990).

We need to ask ourselves if the psychological characteristics which nonagenarians demonstrate are primarily genetic or cultural-or if the two are inextricably linked through behavioural genetics? Do our behaviours, our choices, our family and social context influence and imprint our genes, so that each new generation takes forward behaviours and family cultural influences in a way which to date cannot be measured, but only surmised from our understanding of social networks, experiences and trans-generational influences (Laland et al. 2010; Heijmans et al. 2008; Pembrey et al. 2014).

The insights related by nonagenarians about how they understand their own good quality ageing, resonate with present-day public health campaigns encouraging people to adopt better life-styles and behaviours in order to set a better life course (Thaler and Sunstein 2008; Marteau et al. 2011; Department of Health 2010). They are living examples of how a combination of factors - family genes, behaviours and beliefs and perhaps a healthy dose of good luck - seem to have improved their chance of living longer and with a better quality of life.

The message for everyone is that taking better care of ourselves, our social networks and actively making life-style choices can increase the chance of 'ageing long and ageing well' and improve the longevity dividend for each person and for society (Olansky et al. 2007). 
Acknowledgments We thank the nonagenarians from the BELFAST study who enthusiastically engaged in the Super Vivere and Beyond 90 Together projects and shared their life stories and their views on their healthy ageing with us. The research was funded in part by the Changing Ageing Project, Queens University Belfast Foundation Trust for the project grant.

Open Access This article is distributed under the terms of the Creative Commons Attribution License which permits any use, distribution, and reproduction in any medium, provided the original author(s) and the source are credited.

\section{References}

Al-Jaderi Z, Maghazachi AA (2013) Effects of vitamin D calcipotriol and FTY720 on the expression of surface molecules and cytolytic activities of human natural killer cells and dendritic cells. Toxins 5:1932-1947. doi:10.3390/ toxins5111932

Baltes P, Baltes M (1990) Psychological perspectives on successful aging: the model of selective optimisation with compensation. In: Baltes P, Baltes M (eds) Successful aging: perspectives from the behavioral sciences. Cambridge University Press, New York, pp 1-34

Beekman M, Blanché H, Perola M, Hervonen A, Bezrukov V, Sikora E, GEHA Consortium (2013) Genome-wide linkage analysis for human longevity: genetics of healthy aging study. Aging Cell 2:184-193. doi:10.1111/acel.12039

Bennati E, Murphy A, Cambien F, Whitehead AS, Archbold GP, Young IS, Rea IM (2010) BELFAST centenarians: a case of optimised cardiovascular risk? Curr Pharm Des 16:789-795. doi:10.2174/138161210790883697

Bennet AM, Angelantonio ED, Ye Z, Wensley F, Dahlin A, Ahlbom A, Keavney B, Collins R, Wiman B, Faire U, Danesh J (2007) Association of apolipoprotein E genotypes with lipid levels and coronary risk. JAMA 298:1300-1311. doi:10.1001/jama.298.11.1300

Brooks-Wilson AR (2013) Genetics of healthy aging and longevity. Hum Genet 132:1323-1338. doi:10.1007/ s00439-013-1342-z

Camargo A, Ruano J, Fernandez JM, Parnell LD, Jimenez A, Santos-Gonzalez M, Mari C, Perez-Martinez P, Uceda M, Lopez-Miranda J, Perez-Jimenez F (2010) Gene expression changes in mononuclear cells from patients with metabolic syndrome after acute intake of phenol-rich virgin olive oil. BMC Genomics 11:253. doi:10.1186/1471-2164-11-253

Cantorna MT, Zhao J, Yang L (2012) Vitamin D, invariant natural killer T-cells and experimental autoimmune disease. Proc Nutr Soc 71:62-66. doi:10.1017/S002966511 1003193

Carvalho A, Rea IM, Parimon T, Cusack BJ (2014) Physical activity and cognitive function in individuals over 60 years of age: a systematic review. Clin Interv Aging 9:661-682. doi:10.2147/CIA.S55520

Cauley JA, Eichner JE, Kamboh MI, Ferrell RE, Kuller LH (1993) Apo E allele frequencies in younger (age 42-50) vs older (age 65-90) women. Genet Epidemiol 10:27-34
Chuang YF, Hayden KM, Norton MC, Tschanz J, Breitner JC, Welsh-Bohmer KA, Zandi PP (2010) Association between APOE epsilon4 allele and vascular dementia: the cache county study. Dement Geriatr Cogn Disord 29:248-253. doi:10.1159/000285166

Cobley JN, Moult PR, Burniston JG, Morton JP, Close GL (2014) Exercise improves mitochondrial and redoxregulated stress responses in the elderly: better late than never! Biogerontology. doi:10.1007/s10522-014-9546-8

Corbo RM, Scacchi R (1999) Apolipoprotein E (APOE) allele distribution in the world. Is APOE 4 a 'thrifty' allele? Ann Hum Genet 63:301-310. doi:10.1046/j.1469-1809.1999. 6340301.x

Corder EH, Saunders AM, Strittmatter WJ, Schmechel DE, Gaskell PC, Small GW, Roses AD, Haines JL, PericakVance MA (1993) Gene dose of apolipoprotein E type 4 allele and the risk of Alzheimer's disease in late onset families. Science 261:921-923. doi:10.1126/science. 8346443

Corder EH, Lannfelt L, Viitanen M, Corder LS, Manton KG, Winblad B, Basun H (1996) Apolipoprotein E genotype determines survival in the oldest old (85 years or older) who have good cognition. Arch Neurol 53:418-422. doi:10.1001/archneur.1996.00550050048022

Corella D, Carrasco P, Sorlí JV, Estruch R, Rico-Sanz J, Martínez-González MÁ et al (2013) Mediterranean diet reduces the adverse effect of the TCF7L2-rs7903146 polymorphism on cardiovascular risk factors and stroke incidence: a randomized controlled trial in a high-cardiovascular-risk population. Diabetes Care 36:3803-3811. doi: $10.2337 / \mathrm{dc} 13-0955$

de Lorgeril M, Salen P (2006) The mediterranean-style diet for the prevention of cardiovascular diseases. Public Health Nutr 9(1):118-123. doi:10.1079/PHN2005933

Deelan J, Beekman M, Hae-Won Uh, Helmer Q, Kuningas M, Christiansen L, Kremer D et al (2011) Genome-wide association study identifies a single major locus contributing to survival into old age: the APOE locus revisited. Aging Cell 10:686-698. doi:10.1111/j.1474-9726.2011.00705.x

Deelen J, Beekman M, Uh H-W, Broer L, Ayers KL, Tan Q et al (2014) Genome-wide association meta-analysis of human longevity identifies a novel locus conferring survival beyond 90 years of age. Hum Mol Genet 23:4420-4432. doi: $10.1093 / \mathrm{hmg} / \mathrm{ddu} 139$

Department of Health (2010) White paper: healthy lives, healthy People: our strategy for public health in England, UK. The Stationery Office Limited on behalf of the Controller of Her Majesty's Stationery Office. ISBN 9780101798525

Di Bona D, Vasto S, Capurso C, Christensen L, Deiana L, Franceschi C, Hurme M, Mocchegiani E, Rea M, Lio D, Candore G, Caruso C (2009) Effect of interleukin-6 polymorphisms on human longevity: a systemic review and meta-analysis. Ageing Res Rev 8:36-42. doi:10.1016/j.arr. 2008.09.001

Editor (2015) Time for the Social Sciences. Nature, 517. doi:10. $1038 / 517005 a$

Eggertsen G, Tegelman R, Ericsson S, Angelin B, Berglund L (1993) Apolipoprotein E polymorphism in a healthy Swedish population: variation of allele frequency with age and relation to serum lipid concentrations. Clin Chem $39: 2125-2129$ 
Eichner JE, Dunn ST, Perveen G, Thompson DM, Stewart KE, Stroehla BC (2002) Apolipoprotein E polymorphism and cardiovascular disease: a HuGE review. Am J Epidemiol 155:487-495. doi:10.1093/aje/155.6.487

Ekelund U, Ward HA, Norat T, Luan J, May AM, Weiderpass E (2015) Physical activity and all-cause mortality across levels of overall and abdominal adiposity in European men and women: the European Prospective Investigation into Cancer and Nutrition Study (EPIC). AJCN. doi:10.3945/ ajen.114.100065

Erickson KI, Voss MW, Prakash RS, Basak C, Szabo A, Chaddock L, Kim JS, Heo S, Alves H, White SM, Wojcicki TR, Mailey E, Vieira VJ, Martin SA, Pence BD, Woods JA, McAuley E, Kramer AF (2011) Exercise training increases size of hippocampus and improves memory. PNAS 108:3017-3022. doi:10.1073/pnas.1015950108

Ershler WB (1993) Interleukin-6: a cytokine for gerontologists. J Am Geriatr Soc 41:176-181

Evert J, Lawler E, Bogan H, Perls TJ (2003) Morbidity profiles of centenarians: survivors, delayers and escapers. J Gerontol A 58:M232-M237. doi:10.1093/gerona/58.3. $\mathrm{m} 232$

Folstein MF, Folstein SE, McHugh PR (1975) Mini mental state. A practical method for grading the cognitive state of patients for the clinician. J Psychiatr Res 12:189-198. doi:10. 1016/0022-3956(75)90026-6

Franceschi C, Bonafè M, Valensin S, Olivieri F, De Luca M, Ottaviani E, De Benedictis G (2000) Inflamm-aging. An evolutionary perspective on immunosenescence. Ann N Y Acad Sci 908:244-254. doi:10.1111/j.1749-6632.2000. tb06651.x

Francis DD, Champagne FA, Liu D, Meaney MJ (1999) Maternal care, gene expression, and the development of individual differences in stress reactivity. Ann N Y Acad Sci 896:66-84. doi:10.1111/j.1749-6632.1999.tb08106.x

Frisoni GB, Louhija J, Geroldi C, Trabucchi M (2001) Longevity and the epsilon 2 allele of apolipoprotein E: the finnish centenarians study. J Gerontol A 56:75-78. doi:10. 1093/gerona/56.2.M75

Fung TT, Rexrode KM, Mantzoros CS, Manson JE, Willett WC, $\mathrm{Hu}$ FB (2009) Mediterranean diet and incidence of and mortality from coronary heart disease and stroke in women. Circulation 119:1093-1100. doi:10.1161/ CIRCULATIONAHA.108.816736

Furukawa TA, Ogura A, Hirai T, Fujihara S, Kitamura T, Takahashi K (1999) Early parental separation experiences among patients with bipolar disorder and major depression: a case-control study. J Affect Disord 52:85-91. doi:10. 1016/S0165-0327(98)00054-8

Ganzevoort RR, Bouwer J (2007) Life story methods and care for the elderly: an empirical research project in practical theology. In: Ziebertz H-G, Schweitzer F (eds) Dreaming the land: theologies of resistance and hope., International Academy of Practical Theology, Brisbane 2005LIT, Münster, pp 140-151

Garagnani P, Giuliani C, Pirazzini C, Olivieri F, Bacalini MG, Ostan R, Mari D, Passarino G, Monti D et al (2013) Centenarians as super-controls to assess the biological relevance of genetic risk factors for common age-related diseases: a proof of principle on type 2 diabetes. Aging (Albany NY) 5:373-385 (PMCID: PMC3701112)
Gibney MJ, Walsh MC (2013) The future direction of personalised nutrition: my diet, my phenotype, my genes. Proc Nutr Soc 72:219-225. doi:10.1017/S0029665112003436

Glaser BG, Strauss AL (1967) The discovery of grounded theory: strategies for qualitative research. Aldine De Gruyter, New York. ISBN 978-0-202-30260-7

Glaser JP, Van Os J, Portegijs PJ, Myin-Germeys I (2006) Childhood trauma and emotional reactivity to daily life stress in adult frequent attenders of general practitioners. J Psychosom Res 61:229-236. doi:10.1016/j.jpsychores. 2006.04.014

Hagberg JM, Wilund KR, Ferrell RE (2000) APO E gene and gene-environment effects on plasma lipoprotein-lipid levels. Physiol Genomics 4:101-108

Heijmans BT, Tobi EW, Stein AD, Putter H, Blauw GJ, Susser ES, Slagboom PE, Lumey LH (2008) Persistent epigenetic differences associated with prenatal exposure to famine in humans. PNAS 105:17046-17049. doi:10.1073/pnas. 0806560105

Hogue CW Jr, Stearns JD, Colantuoni E, Robinson KA, Stierer T, Mitter N, Pronovost PJ, Needham D (2009) The impact of obesity on outcomes after critical illness: a meta-analysis. Intensiv Care Med 35:1152-1170. doi:10.1007/ s00134-009-1424-5

Ilveskoski E, Perola M, Lehtimaki T, Laippala P, Savolainen V, Pajarinen J, Penttila A, Lalu KH, Mannikko A, Liesto KK, Koivula T, Karhunen PJ (1999) Age-dependent association of apolipoprotein E genotype with coronary and aortic atherosclerosis in middle-aged men: an autopsy study. Circulation 6:608-613. doi:10.1161/01.CIR.100.6.608

Jian-Gang Z, Yong-Xing M, Chuan-Fu W, Pei-Fang L, SongBai Z, Nui-Fan G, Guo-Yin F, Lin H (1998) Apolipoprotein $\mathrm{E}$ and longevity among Han Chinese population. Mech Ageing Dev 104:159-167. doi:10.1016/S0047-6374(98) 00067-0

Kervinen K, Savolainen MJ, Salokannel J, Hynninen A, Heikkinen J, Ehnholm C, Koistinen MJ, Kesäniemi YA (1994) Apolipoprotein E and B polymorphismslongevity factors assessed in nonagenarians. Atherosclerosis 105:89-95

Kuperman EF, Showalter JW, Lehman EB, Leib AE, Kraschnewski JL (2013) The impact of obesity on sepsis mortality: a retrospective review. BMC Infect Dis 13:377. doi:10.1186/1471-2334-13-377

Laland KN, Odling-Smee J, Myles S (2010) How culture shaped the human genome: bridging genetics and the human sciences together. Nat Rev Genet 11:137-148. doi:10.1038/ $\operatorname{nrg} 2734$

Lamina S, Ezema CI, Theresa AI, Anthonia EU (2013) Effects of free radicals and antioxidants on exercise performance. Oxid Antioxid Med Sci 2:83-91. doi:10.5455/oams. 010413.rv.005

Lawrence $\mathrm{T}$ (2009) The nuclear factor NF- $\kappa \mathrm{B}$ pathway in inflammation. Cold Spring Harb Perspect Biol 1:a001651. doi:10.1101/cshperspect.a001651

Licastro F, Porcellini E, Caruso C, Lio D, Corder EH (2007) Genetic risk profiles for Alzheimer's disease: integration of APOE genotype and variants that up-regulate inflammation. Neurobiol Aging 28:1637-1643

Lightart GJ, Corberand JX, Fornier C, Galanaud P, Hijmans W, Kennes B, Muller-Hermelink HK, Steinman GG (1984) 
Admission criteria for immunogerontological studies in man: the SENIEUR protocol. Mech Ageing Dev 28:47-55

Lio D, Marino V, Serauto A, Gioia V, Scola L, Crivello A, Forte GI, Colonna-Romano G, Candore G, Caruso C (2002) Genotype frequencies of the $+874 \mathrm{~T} \mathrm{~T}$ A single nucleotide polymorphism in the first intron of the interferon- $\gamma$ gene in a sample of Sicilian patients affected by tuberculosis. Eur J Immunogenet 29:371-374. doi:10.1046/j.1365-2370.2002. 00327.x

Lojovich JM (2010) The relationship between aerobic exercise and cognition: is movement medicinal? J Head Trauma Rehabil 25:184-192. doi:10.1097/HTR.0b013e3181dc $78 \mathrm{~cd}$

Lu F, Guan H, Gong B, Liu X, Zhu R, Wang Y, Qian J, Zhou T, Lan X, Wang P, Lin Y, Ma S, Lin H, Zhu Z, Chen R, Zhu X, Shi Y, Yang Z (2014) Genetic variants in PVRL2TOMM40-APOE region are associated with human longevity in a Han Chinese population. PLoS One 9(6):e99580. doi:10.1371/journal.pone.0099580

Lushchak VI (2014) Dissection of the hormetic curve: analysis of components and mechanisms. Dose Response 12:466-479. doi:10.2203/dose-response.13-051

Mariani E, Ravaglia G, Forti P, Meneghetti A, Tarozzi A, Maioli F, Boschi F, Pratelli L, Pizzoferrato A, Piras F, Facchini A (1999) Vitamin D, thyroid hormones and muscle mass influence natural killer (NK) innate immunity in healthy nonagenarians and centenarians. Clin Exp Immunol 116:19-27. doi:10.1046/j.1365-2249.1999.00855.x

Marteau TM, Ogilvie D, Roland M, Suhrcke M, Kelly MP (2011) Judging nudging: can nudging improve population health? BMJ 342:d228. doi:10.1136/bmj.d228

Masson LF, McNeill G, Avenell A (2003) Genetic variation and the lipid response to dietary intervention: a systematic review. Am J Clin Nutr 77:1098-1111

Mattson MP (2012) Evolutionary aspects of human exercise. Ageing Res Rev 11:347-352. doi:10.1016/j.arr.2012.01. 007

Maxwell LD, Ross OA, Curran MD, Rea IM, Middleton D (2004) Investigation of KIR diversity in immunosenescence and longevity within the Irish population. Exp Gerontol 39:1223-1232. doi:10.1016/j.exger.2004.05.003

McKellar G, Morrison E, McEntegart A, Hampson R, Tierney A, Mackle G, Scoular J, Scott JA, Capell HA (2007) A pilot study of a Mediterranean-type diet intervention in female patients with rheumatoid arthritis living in areas of social deprivation in Glasgow. Ann Rheum Dis 66:1239-1243. doi:10.1136/ard.2006.065151

McNerlan SE, Rea IM, Alexander HD, Morris TCM (1998) Changes in natural killer cells, the CD8 + CD57 + subset and related cytokines in healthy aging. J Clin Immunol 18:31-38. doi:10.1023/A:1023283719877

McNerlan SE, Armstrong MA, Ross OA, Rea IM (2009) Cytokine expression and production changes in very old age. In: Fulop T, Franceschi C, Hirokawa K, Pawelec G (eds) Handbook of immunosenesence: basic understanding and clinical applications, vol 40. Springer, Berlin, pp 771-785. ISBN 978-1-4020-9063-9

Meaney MJ (2001) Maternal care, gene expression, and the transmission of individual differences in stress reactivity across generations. Annu Rev Neurosci 24:1161-1192. doi:10.1146/annurev.neuro.24.1.1161
Meaney MJ, Szyf M (2005) Environmental programming of stress responses through DNA methylation: life at the interface between a dynamic environment and a fixed genome. Dialogues Clin Neurosci 7:103-123 (PMCID: PMC3181727)

Mena MP, Sacanella E, Vazquez-Agell M, Morales M, Fitó M, Escoda R, Serrano-Martínez M, Salas-Salvadó J, Benages N, Casas R, Lamuela- Raventós RM, Masanes F, Ros E, Estruch R (2009) Inhibition of circulating immune cell activation: a molecular anti-inflammatory effect of the Mediterranean diet. Am J Clin Nutr 89:248-256. doi:10. 3945/ajcn.2008.26094

Merkley CM, Jian C, Mosa A, Tan Y-F, Wojtowicz JM (2014) Homeostatic regulation of adult hippocampal neurogenesis in aging rats: long-term effects of early exercise. Front Neurosci 8:174. doi:10.3389/fnins.2014.00174

Miyaji C, Watanabe H, Minagawa M, Toma H, Kawamura T, Nohara Y, Nozaki H, Sato Y, Abo T (1997) Numerical and functional characteristics of lymphocyte subsets in centenarians. J Clin Immunol 5:420-429

Mocchegiani E, Malavolta M (2004) NK and NKT cell functions in immunosenescence. Aging Cell 3:177-184. doi:10. 1111/j.1474-9728.2004.00107.x

Moore SC, Patel AV, Matthews CE, Berrington de Gonzalez A, Park Y et al (2012) Leisure time physical activity of moderate to vigorous intensity and mortality: a large pooled cohort analysis. PLoS Med 9(11):e1001335. doi:10. 1371/journal.pmed.1001335

Muscari A, Giannoni C, Pierpaoli L, Berzigotti A, Maietta P, Foschi E, Ravaioli C, Poggiopollini G, Bianchi G, Magalotti D, Tentoni C, Zoli M (2010) Chronic endurance exercise training prevents aging-related cognitive decline in healthy older adults: a randomized controlled trial. Int J Geriatr Psychiatry 10:1055-1064. doi:10.1002/gps.2462

Neeper SA, Gómez-Pinilla F, Choi J, Cotman C (1995) Exercise and brain neurotrophins. Nature 373:109

Northern Arizona University (1998) In: Chapter 15 promote social interaction and friendships: On-LineLesson. http:// www.prm.nau.edu/prm326/promote_social_inter_lesson. htm. Accessed 1 March 2015

Olansky SJ, Perry D, Miller RA, Butler RN (2007) Pursuing the longevity dividend: scientific goals for an aging world. Ann N Y Acad Sci 1114:11-13. doi:10.1196/annals.1396.050

Ordovas JM (2008) Genotype-phenotype associations: modulation by diet and obesity. Obesity (Silver Spring) 16(Suppl 3):S40-S46. doi:10.1038/oby.2008.515

Pang TYC, Hannan AJ (2013) Enhancement of cognitive function in models of brain disease through environmental enrichment and physical activity. Neuropharm 64:515-528. doi:10.1016/j.neuropharm.2012.06.029

Patten AR, Sickmann H, Hryciw BN, Kucharsky T, Parton R, Kernick A, Christie BR (2013) Long-term exercise is needed to enhance synaptic plasticity in the hippocampus. Learn Mem 20:642-647. doi:10.1101/lm.030635.113

Pembrey M, Saffery R, Bygren LO (2014) Network in epigenetic epidemiology: human transgenerational responses to early-life experience: potential impact on development, health and biomedical research. J Med Genet 51:563-572. doi:10.1136/jmedgenet-2014-102577

Peralbo E, Alonso C, Solana R (2007) Invariant NKT and NKTlike lymphocytes:two different $\mathrm{T}$ cell subsets that are 
differentially affected by ageing. Exp Gerontol 42:703-708. doi:10.1016/j.exger.2007.05.002

Perls T (2002) Genetic and environmental influences on exceptional longevity and the AGE nomogram. Ann N Y Acad Sci 959:1-13

Ploughman M (2008) Exercise is brain food: the effects of physical activity on cognitive function. Dev Neurorehabil 11:236-240. doi:10.1080/17518420801997007

Radak Z, Chung HY, Goto S (2005) Exercise and hormesis: oxidative stress-related adaptation for successful aging. Biogerontology 6:71-75. doi:10.1007/s10522-004-7386-7

Radak Z, Chung HY, Koltai E, Taylor AW, Goto S (2008) Exercise, oxidative stress and hormesis. Ageing Res Rev 7:34-42. doi:10.1016/j.arr.2007.04.004

Raichlen DA, Alexander GE (2014) Exercise, APOE genotype, and the evolution of the human lifespan. TINS 37:247-255. doi:10.1016/j.tins.2014.03.001

Ravaglia G, Forti P, Maioli F, Bastagli L, Facchini A, Mariani E, Savarino L, Sassi S, Cucinotta D, Lenaz G (2000) Effect of micronutrient status on natural killer cell immune function in healthy free-living subjects aged $\geq 90 \mathrm{y} 1-3$. Am J Clin Nutr 71:590-598

Rea Maeve (2013) Living long and ageing well: Insights from nonagenarians. In: Davidson S, Goodwin J, Rossall P (eds) Improving later life: understanding the oldest old age, UK, pp 74-77. ISBN 978-0-9568731-6-3. http://www.ageuk. org.uk/Documents/EN-GB/For-professionals/Research/ Improving \%20Later\%20Life \%202\%20WEB.pdf?dtrk= true

Rea M, Rea S (2011) Super vivere: reflections on long life and ageing well. Blackstaff Press, Belfast. ISBN 978-0-85640866-3

Rea IM, McDowell I, McMaster D, Smye M, Stout R, Evans A, MONICA Group (Belfast) (2001) Apolipoprotein E alleles in nonagenarian subjects in the Belfast Elderly Longitudinal Free-Living Ageing STudy (BELFAST). Mech Ageing Dev 122:1367-1372. doi:10.1016/S0047-6374(01) 00278-0

Rea IM, Ross OA, Armstrong M, McNerlan S, Alexander HD, Curran MD, Middleton D (2003) Interleukin-6-gene C/G 174 polymorphism in nonagenarian and octogenarian subjects in the BELFAST study. Reciprocal effects on IL6, soluble IL-6 receptor and for IL-10 in serum and monocyte supernatants. Mech Ageing Dev 124:555-561. doi:10.1016/S0047-6374(03)00036-8

Rea IM, Candore G, Cavallone L, Olivieri F, Cardelli M, Franceschi C, Colonna-Romano G, Lio D, Ross OA, Caruso C (2006) Longevity. In: Vandenbroeck K (ed) Cytokine gene polymorphisms in multifactorial conditions, vol 29. CRC Press, Taylor \& Francis, Boca Raton, pp 379-391

Rea IM, Myint PK, Mueller H, Murphy A, Archbold PR, McNulty H, Patterson CC (2009) Nature and nurture: BMI and blood pressure at 90 . Findings from the Belfast Elderly Longitudinal Free-Living Ageing STudy (BELFAST). AGE 31:261-267. doi:10.1007/s11357-009-9096-1

Rea IM, Maxwell LD, McNerlan SE, Alexander HD, Curran MD, Middleton D, Ross OA (2013) Killer immunoglobulin-like receptors (KIR) haplogroups A and B track with natural killer cells and cytokine profile in aged subjects: observations from octo/nonagenarians in the Belfast Elderly Longitudinal Free-living Aging Study (BELFAST). Immun Ageing 10:35. doi:10.1186/17424933-10-35

Rentoul L (1997) Developmental problems in later life. In: Norman IJ, Redfern SJ (eds) Mental health care for elderly people. Churchill Livingstone, New York, pp 69-88

Rhyu IJ, Bytheway JA, Kohler SJ, Lange H, Lee KJ, Boklewski J, McCormick K, Williams NI, Stanton GB, Greenough WT, Cameron JL (2010) Effects of aerobic exercise training on cognitive function and cortical vascularity in monkeys. Neuroscience 167:1239-1248. doi:10.1016/j. neuroscience.2010.03.003

Ross OA, Curran MD, Meenagh A, Williams F, Barnett YA, Middleton D, Rea IM (2003) Study of age-association with cytokine gene polymorphisms in an aged Irish population. Mech Ageing Dev 124:199-206. doi:10.1016/S00476374(02)00132-X

Rowe JW, Kahn RL (1997) Successful aging. Gerontologist 37:433-440

Sansoni P, Cossarizza A, Brianti V, Fagnoni F, Snelli G, Monti D, Marcato A, Passeri G, Ortolani C, Forti E (1993) Lymphocyte subsets and natural killer cell activity in healthy old people and centenarians. Blood 82:2767-2773

Schachter F, Faure-Delenauf L, Guenot F, Rouger H, Froguet P, Lesueur- Ginot L, Cohen D (1994) Genetic associations with human longevity at the ApoE and ACE loci. Nat Genet 6:29-32. doi:10.1038/ng0194-29

Skoldstam L, HagfoLs L, Johansson G (2003) An experimental study of a Mediterranean diet intervention for patients with rheumatoid arthritis. Ann Rheum Dis 62:208-214. doi:10. 1136/ard.62.3.208

Spoto B, Mattace-Raso F, Sijbrands E, Leonardis D, Testa A, Pisano A, Pizzini P, Cutrupi S, Parlongo RM, D'Arrigo G, Tripepi G, Mallamaci F, Zoccali C (2014) Association of IL-6 and a functional polymorphism in the IL-6 gene with cardiovascular events in patients with CKD. Clin J Am Soc Nephrol. doi:10.2215/CJN. 07000714

Strauss A, Corbin J (1990) Basics of qualitative research: grounded theory procedures and techniques. Sage Publications Inc, Newbury Park

Thaler RH, Sunstein CS (2008) Nudge: improving decisions about health, wealth, and happiness. Yale New Haven Press, New Haven. ISBN 9780300122237

Vaynmana SS, Yinga Z, Yina D, Gomez-Pinilla F (2006) Exercise differentially regulates synaptic proteins associated to the function of BDNF. Brain Res 1070:124-130. doi:10. 1016/j.brainres.2005.11.062

von Essen MR, Kongsbak M, Schjerling P, Olgaard K, Odum N, Geisler C (2010) Vitamin D controls T cell antigen receptor signalling and activation of human T cells. Nat Immunol 11:344-349. doi:10.1038/ni.1851

Wen CP, Wai JPM, Tsai MK, Yang YC, Cheng TYD, Lee M-C, Chan HT, Tsao CK, Tsai S-P, Wu X (2011) Minimum amount of physical activity for reduced mortality and extended life expectancy: a prospective cohort study. Lancet 378:1244-1253. doi:10.1016/S0140-6736(11)60749-6 
Yates LB, Djousse L, Kurth T, Buring JE, Gaziano M (2008) Exceptional longevity in men: modificable factors associated with survival and function to age 90 years. Arch Intern Med 168:284-290. doi:10.1001/archinternmed.2007.77
Zhang F, Lewis M, Yang G, Iriondo-Perez J, Zeng Y, Liu J (2008) Apolipoprotein E polymorphism, life stress and self-reported health among older adults. J Epidemiol Community Health 62:e3. doi:10.1136/jech.2007.063917 